\title{
Étude comparative de quelques fertilisants (Bat-guano et DAP) sur le rendement du niébé (Vigna unguiculata, $L$. Walp.) dans la région de Gandajika (RDC)
}

\author{
Michel Nkongolo Mulambuila1, Robert Mukendi Kamambo², Carcy Tshimbombo Jadika², J. Michel \\ Mutombo Tshibamba', Moïse Kalambaie Binm Mukanya183. \\ 1 : Université Officielle de Mbujimayi, Faculté des Sciences Agronomiques, Mbujimayi, Kasaï-Oriental, RD. Congo. \\ 2 : Institut National pour l'Étude et Recherches Agronomiques « INERA »/Gandajika, Kasaï-Oriental, RD. Congo. \\ 3 : Université Pédagogique Nationale « UPN »/Kinshasa, RD. Congo. \\ Contact: +243815060834; email: michel.nkongolo2005@gmail.com; jeanmichelmutombo@gmail.com
}

Original submitted in on $2^{\text {nd }}$ July 2015. Published online at www.m.elewa.org on $31^{\text {st }}$ August 2015

http://dx.doi.org/10.4314/iab.v92i1.9

\section{RÉSUME}

Objectif : En Afrique Sub-saharienne, plus particulièrement dans la région de Gandajika, en RD. Congo, la culture de niébé revêt une grande importance car la consommation de cette denrée s'inscrit dans la lutte contre la malnutrition protéino-calorique. Au regard de sa richesse en protéine, le niébé peut servir d'un substitut aux produits carnés qui ne sont pas à la portée de la grande partie des habitants dans cette région. Cependant, sa production fait face à quelques contraintes notamment l'état de fertilité des sols en ce qui concerne l'élément phosphore surtout, qui joue un rôle prépondérant dans le développement des légumineuses dont le niébé. Cette étude a comme objectif de : (1) comparer ces deux formes d'apport du Bat-guano (dissoute et non dissoute) pour déterminer la plus efficace, en supposant que la dissoute libérerait rapidement et suffisamment les éléments nutritifs ; (2) comparer ensuite ces deux formes de à l'engrais minéral (DAP) ; (3) comparer enfin l'association de Bat-guano dissout avec le DAP à celle de Batguano non dissout +DAP dans une gestion intégrée de la fertilité du sol (GIFS) sur la culture de niébé. Méthodologie et résultats : Pour atteindre ces objectifs, 6 traitements ont été formulés sous un dispositif en blocs complets randomisés avec trois répétitions. A l'issue de cette étude, les résultats ci-après, ont été enregistrés. Concernant les deux formes de bat-guano, la forme dissoute a donnée un rendement de 0.916 t.ha $^{-1}$ tandis que la forme non dissout 0.730 t.ha $^{-1}$, l'analyse de la variance n'a pas émis de différence observée arithmétiquement, 0.186 t.ha- $^{-1}$, soit $186 \mathrm{Kg} . h a-1^{-1}$. Par conséquent, les deux formes ont eu le même effet sur le rendement de la culture de niébé, c'est-à-dire que l'une peut être utilisée à la place de l'autre. Les deux formes de bat-guano face à l'engrais minéral (DAP), ce dernier a donné un rendement de 0.780 tha- $^{-1}$, l'analyse de la variance n'a pas dégagé de différence significative, cependant, arithmétiquement, la forme dissoute du bat-guano à produit plus que l'engrais minéral, soit une différence de 0,136 t.ha-1 $^{-1}$ ou $136 \mathrm{~kg}_{\text {.ha-1 }}$. Ces deux formes ont eu le même effet sur le rendement de la culture de niébé. L'association de ces deux formes à l'engrais minéral produit les mêmes effets que les autres traitements sur la culture de niébé soit 0,840 tha- $^{-1}$ pour l'association bat-guano dissout et engrais minéral (DAP) et 0,760 tonne/ha pour l'association bat-guano non dissout plus DAP. 
Conclusion et application des résultats : Dans le cadre de cette étude, le Bat-guano peut être recommandé aux paysans producteurs étant donné sa richesse en éléments nutritifs, son accessibilité, son coût moins onéreux comparativement aux engrais minéraux (DAP). La contrainte de sa disponibilité est contournée par l'élevage de Chauve-souris qui produisent suffisamment ce fertilisant. En rapport, la GIFS, bien que le facteur eau n'ait pas permis au Bat-guano d'enregistrer les résultats escomptés. Son utilisation peut bien se justifier pour minimiser la quantité d'engrais minéraux à utiliser.

Mots clés : Comparaison, bat-guano, diammonium de phosphate, rendement, niébé, Gandajika, RDC

\section{ABSTRACT \\ Comparative study of some fertilizers (Bat-guano and DAP) Performance of cowpea (Vigna unguiculata L. Walp.) In the Gandajika region (DRC)}

Objective: In Sub-Saharan Africa, particularly in the area Gandajika, DR. Congo, the culture of cowpea is important because consumption of this commodity is in the fight against protein-calorie malnutrition. Given its high protein, cowpeas can be used as a substitute for meat products which are not within the reach of much of the population in this region. However, its production faces several constraints including soil fertility status regarding the element especially phosphorus, which plays a major role in the development of legumes including cowpea. This study aims to: (1) compare the two forms of the Bat-guano intake (dissolved and undissolved) to determine the most effective, assuming that the dissolved quickly and free up enough nutrients; (2) then compare the two forms of the mineral fertilizer (DAP); (3) Finally, comparing the combination of Bat-guano dissolves with DAP than Bat-guano undissolved + DAP in integrated management of soil fertility (GIFS) on the culture of cowpea.

Methodology and results: To achieve these goals, six treatments were formulated in a device randomized complete block design with three replications. At the end of this study, the following results were recorded:

Regarding the two forms of bat guano, dissolved form has given a yield of $0.916 \mathrm{t}$ ha- 1 while the shape undissolved $0.730 \mathrm{t}$ ha-1, variance analysis has not issued any observed difference arithmetically, $0.186 \mathrm{t}$ ha-1 or 186 kg.ha-1. Therefore, the two forms had the same effect on the crop yield of cowpea, that is to say that one can be used in place of the other. Both forms of bat guano facing mineral fertilizer (DAP), the latter gave a yield of $0.780 \mathrm{t}$ ha-1, analysis of variance has not reached significant difference, however, arithmetically, dissolved form of bat guano produced more than mineral fertilizer, a difference of $0.136 \mathrm{t}$ ha1 and $136 \mathrm{~kg}$.ha-1. Both forms have the same effect on the crop yield of cowpea The combination of these two forms of mineral fertilizers produced the same effects than other treatments on the culture of cowpea is $0.840 \mathrm{t} / \mathrm{ha}-1$ for the association bat guano and dissolves mineral fertilizer (DAP) and 0.760 ton / ha for the association bat guano undissolved more DAP.

Conclusion and application of results: As part of this study, the Bat-guano can be recommended to peasant producers given its richness in nutrients, its accessibility, its cheaper cost compared to mineral fertilizer (DAP). The availability constraint is bypassed by the Bat breeding that produce enough fertilizer this. In the report, ISFM, although the water factor has not enabled the Bat guano-record the desired results. Its use may well be justified to minimize the amount of mineral fertilizers to use.

Keywords: Comparison, bat guano, diammonium phosphate, yield, cowpea, Gandajika, DRC

\section{INTRODUCTION}

En Afrique sub-saharienne, la culture de légumineuses, singulièrement celle de niébé (Vigna unguiculata, L., Walp.) revêt une grande importance car la consommation de cette denrée s'inscrit bien dans la lutte contre l'insécurité alimentaire. Celle-ci se caractérise essentiellement par la malnutrition protéino-calorique et le niébé, de par sa teneur en protéine, peut constituer un substitut aux produits carnés qui ne sont pas à la portée de la plus grande partie des habitants dans cette partie du monde. Cependant, la production du niébé, rencontre quelques contraintes 
notamment l'état de fertilité des sols en ce qui concerne la situation de l'élément phosphore, pourtant jouant un rôle important dans le développement de cette culture (Falisse $H_{\text {., }}$ et Lambert J., 1995). Les ferrasols sont des sols dominants dans les régions tropicales d'Afrique et d'Amérique. Ils couvrent une grande superficie en République Démocratique du Congo, tel que celui de Gandajika. Ce type de sols se caractérise par une faible capacité d'échange cationique, une forte concentration d'oxydes de fer et d'hydroxydes d'aluminium qui sont responsables de la fixation du phosphore le rendant ainsi indisponible aux plants de culture (Bui et Al., 1989 ; Bartoli et al., 1992; Sanchez et Logan, 1992; Buresh et al., 1997 ; Decker, 1999 ; Neufeldt,1999). Ce type de sols a une faible rétention en eau, cela est due à la présence d'une grande macroporosité liée à la microstructure stable résultant d'oxyhydroxydes chargés positivement et de Kaolinite mélangée à la matière organique chargé négativement (Bui et Al., 1989 ; Bartoli et al, 1992). Cette situation a comme conséquence, la carence en eau dont souffrent les plantes pendant les périodes sèches de la saison de pluie, malgré des teneurs en argile élevées (Neufeldts, 1999). Ce type de sols se caractérise aussi la faible teneur en matière organique due une décomposition rapide de la matière végétale suite aux précipitations fréquentes (régions tropicales et subtropicales). Cet état de chose (faible fertilité) de ces sols exige une meilleure gestion de la fertilité par de fertilisation ou le chaulage permanente. C'est dans cette perspective que, la gestion intégrée de la fertilité du sol (combinaison de la fumure organique et des engrais inorganiques) est impérative, celle-ci fait appelle à plusieurs méthodes déjà mis au point, telle que : L'introduction d'arbres fixateurs d'azote atmosphérique dans le système de cultures en couloir, en agroforesterie (Kang et al., 1984; Young, 1987 ; Ingram, 1990 cité par Muyayabantu, 2013) l'association de cultures. II est bien établi que l'application des engrais chimiques permet la mise rapide à la disposition des cultures des éléments nutritifs, ce qui amène à des rendements élevés. Mais cependant l'utilisation des engrais chimiques dans plusieurs parties de l'Afrique subsaharienne, particulièrement à Gandajika, dans la région centrale de la République Démocratique du Congo; est confrontée à plusieurs contraintes parmi lesquelles, le manque d'investissement et de crédit financier qui en sont d'ordre socioéconomique (Bado et al, 2007 cité par Muyayabantu, 2013). L'utilisation continue des engrais chimiques avec l'absence de restitution des résidus de récolte contribue à augmenter la lixiviation, l'acidification des sols et à la diminution significative de bases échangeables du sol. Ceci équivaut à l'application des engrais chimiques sans amendement organique (Wong et al., 1995 ; Bayayoko et al., 1996; Bationo et al., 1997 ; Coulibaly et al.,1998; Nziguheba et al., 1998). L'utilisation de la fumure organique seule, ne peut soutenir les cultures à cause de l'insuffisance de leur quantité et de leurs niveaux relativement bas en éléments nutritifs (Palm et al., 1997). C'est dans cette optique que cette étude a été menée dans la région de Gandajika dans l'objectif de : (1) comparer ces deux formes d'apport du Bat-guano (dissoute et non dissoute) pour déterminer la plus efficace, en supposant que la dissoute libérerait rapidement et suffisamment les éléments nutritifs ; (2) comparer ensuite ces deux formes de à l'engrais minéral (DAP); (3) comparer enfin l'association de Bat-guano dissout avec le DAP à celle de Bat-guano non dissout +DAP dans une gestion intégrée de la fertilité du sol (GIFS) sur la culture de niébé. Le Bat-guano désigne les excréments de chauve-souris qui sont utilisé comme engrais organique riche en $\mathrm{P}_{2} \mathrm{O}_{5}$ (peroxyde de phosphore) et en Azote. Cependant le DAP est le Diammonium de Phospahte $\left(\mathrm{NH}_{4}\right)_{2} \mathrm{HPO}_{4}$ contenant $21,21 \%$ d'azote et $23,4 \%$ de Phosphore, utilisé comme engrais minéral binaire sur les céréales et les légumineuses. L'apport d'éléments nutritif provenant des engrais organique qui est lent et insuffisant peut être complété par celui des engrais inorganiques qui libère rapidement ces éléments qui sont utilisés par les cultures. L'apport de 1èrs compense celui des seconds (Ayoola et Makinde, 2008) cité par Muyayabantu, 2013. 
MATÉRIELS ET MÉTHODES

Milieu d'étude : Le site de la station de l'INERA de Gandajika a servi de cadre expérimental pour l'étude sous examen. Cette station est située à $7 \mathrm{~km}$ de la cité de Gandajika, dans le territoire portant le même nom, dont les coordonnées géographiques sont $6^{\circ} 43^{\prime} 32,6^{\prime \prime}$ de latitude Sud et $23^{\circ} 56^{\prime} 33,5^{\prime \prime}$ de longitude Est, à 793 $\mathrm{m}$ d'altitude moyenne (Anonyme, 2003). C'est une zone à vocation agricole par excellence pour le Kasaï Oriental.

Sol de Gandajika : Les sols de Gandajika sont formés d'un recouvrement sableux sur un sédiment argileux qui repose, souvent, à faible profondeur, sur une ancienne dalle latéritique (Bado, 1993). La fraction argileuse, peu importante, ne semble pas uniquement constituée de kaolinite. Ces sols contiennent 21 à $23 \%$ d'éléments fins (Culot et Laudelout, 1959). Ils sont, de manière générale, profonds, avec un profil qui décrit la présence de tous les horizons et leurs subdivisions, à l'exception de certains endroits, où, il peut exister une nappe phréatique superficielle.

Climat de Gandajika : Le climat du territoire est du type tropical, $A W_{4}$, selon la classification de Köppen. II se caractérise par l'alternance de deux saisons climatiques, la pluvieuse et la sèche. La 1ère se subdivise en deux, une grande, dite saison $A$, allant du 15août au 31 décembre et une petite, dite saison $B$, qui va du 15 janvier au 15 mai. Quant à la saison sèche, aussi subdivisée en deux, elle va du 31 décembre au 15 janvier, c'est la petite saison sèche, et du 15 mai au 15 août, c'est la grande saison sèche. La moyenne des précipitations annuelles, enregistrées dans la période de 1931 à 1970, est de 1425,8 mm, à la Station de Recherche de I'INERA/Gandajika, et l'humidité atmosphérique relative moyenne est de $72,6 \%$. La température annuelle de l'air varie de $25^{\circ} \mathrm{C}$ dans le nord à $22,5^{\circ} \mathrm{C}$ dans le sud (Anonyme, 1998), soit une moyenne de $23.3^{\circ} \mathrm{C}$. Selon les moyennes observées de 1957 à 1970, au sol, la température moyenne est, au maximum, de $28,2^{\circ} \mathrm{C}$ pendant le mois d'avril qui est le plus chaud, et, au minimum, de $24,4^{\circ} \mathrm{C}$, en juillet qui est le mois le plus froid, à la profondeur de $50 \mathrm{~cm}$. La durée de l'insolation moyenne est de 2400 heures/an (Janssens, 1998). Pendant la durée de l'essai, les valeurs des paramètres observés sont consignées dans le tableau 1.

Tableau 1 : Paramètres climatiques observés pendant l'essai

\begin{tabular}{|c|c|c|c|c|c|}
\hline \multirow{2}{*}{ Mois } & \multicolumn{3}{|c|}{ Température $\left({ }^{\circ} \mathrm{C}\right)$} & Humidité relative (\%) & Précipitations \\
\hline & Max & Min & Moyenne & Moyenne & (mm) \\
\hline Octobre & 305 & 22 & 26,25 & 75,8 & 126,1 \\
\hline Novembre & 31,1 & 19,8 & 25,45 & 78,98 & 179,3 \\
\hline Décembre & 30,1 & 19,5 & 24,8 & 80,40 & 477,6 \\
\hline Janvier & 30,1 & 20,1 & 25,6 & 75,4 & 62,2 \\
\hline
\end{tabular}

Source : station Météo /INERA/Gandajika

Relief et végétation de Gandajika : Le relief de Gandajika est dominé par les plaines, à l'exception de quelques montagnes et plateaux. Comme le climat, il confère à cette région des bonnes potentialités agricoles. La végétation type de Ngandajika est la savane herbeuse boisée (Rishirumuhirwa et al., 1989 ; Anonyme, 1998). Elle est dominée par les Poacées qui couvrent plus de $70 \%$ de la superficie par $\mathrm{m}^{2}$. Les espèces dominantes sont Imperata cylindrica sur les sols lourds et Hyparrhenya dissoluta, Digitaria brazzol, Triunfetta mustersu, Eriosema griseu, Mimosa pudica sporadiquement quelques espèces de la famille de légumineuses comme Mucuna sp, Stylosanthes sp, rencontrés dans les bas-fonds sur sols légers. A l'instar d'autres savanes boisées, on trouve de galeries forestières le long des rivières et ruisseaux.
Matériel biologique : Le matériel biologique utilisé dans cet essai était constitué de semences des niébé (Vigna unguiculata) Variété diamant provenant de I'INERA/Gandajika. C'est une variété de cycle végétatif de 75 jours, qui fleurit à $45 j$ jours. Couleur de gousse à maturité : jaune claire. Couleur de graine: péricarpe bicolore, endocarpe ivoire, couleur hile blanche. Tolérance à Allectra vogelii (INERA, 2007). Elle a le rendement de $1200-1600 \mathrm{~kg}$ par hectare en milieu contrôlé et $500-600 \mathrm{~kg} / \mathrm{kg}$ en milieu paysan.

Matériels fertilisants : Le matériel fertilisant utilisé était le bat-guano, le Diammonium de phosphore (DAP). Le bat-guano se présentait sous deux formes: formes dissoute et non dissoute. Le bat-guano a été épandu avant le semis du niébé tandis que celui-ci a eu lieu 4 semaines après l'épandage de ce fertilisant.

\section{Méthodes}


Dispositif expérimental : Cet essai a été installé selon un dispositif en blocs randomisés complets avec trois répétitions et six unités expérimentales par bloc. Le terrain expérimental avait une superficie de $287 \mathrm{~m}^{2}$ soit $(20,5 \mathrm{mx} 14 \mathrm{~m})$. II a été divisé en 18 parcelles de $12 m^{2}(4 m \times 3 m)$ chacune. Les parcelles étaient séparées par des allées de 0,5m et les blocs séparés de $1 \mathrm{~m}$. Les traitements comparés étaient respectivement :

$$
\begin{aligned}
& >\quad \mathrm{T}_{0}=\text { témoin sans fertilisant } \\
& \mathrm{T}_{1}=\mathrm{BD} \text { (bat-guano dissous, } 6 \mathrm{~kg} \text { dans } 6 \text { litres } \\
& \text { d'eau. } \left.12 \mathrm{~m}^{-2}\right) \text { soit } 5 \text { tonnes dans } 50 \mathrm{hl} \text { d'eau/hectare } \\
& \left.>\quad \mathrm{T}_{2}=\mathrm{BND} \text { (bat-guano non dissous, } 6 \mathrm{~kg} \cdot 12 \mathrm{~m}^{-2}\right) \\
& \text { soit } 5 \text { tonnes } / \text { hectare } \\
& >\quad \mathrm{T}_{3}=\mathrm{DAP}\left(67,21 \mathrm{~kg} \mathrm{~N} \text { et } 23,42 \mathrm{~kg} \mathrm{P} \mathrm{O}_{5} \cdot \mathrm{ha}^{-1}\right. \\
& >\quad \mathrm{T} 4=\left(\frac{B D+D A P}{2}\right)=(2.5 \text { tonnes } \mathrm{BD}+33.6 \mathrm{~kg} \mathrm{~N} \\
& \left.+11,74 \mathrm{~kg} \mathrm{P} \mathrm{P}_{2} \mathrm{O}_{5} \cdot \mathrm{ha}^{-1}\right) \\
& >\quad \mathrm{T} 5=\left(\frac{B N D+D A P}{2}\right)(2.5 \text { tonnes } \mathrm{BND}+33,6 \mathrm{~kg} \mathrm{~N}
\end{aligned}
$$$$
\text { et } 11,74 \mathrm{~kg} \mathrm{P}_{2} \mathrm{O}_{5} / \mathrm{ha} \text { ) }
$$

Itinéraire technique de l'essai : Étant donné le retard de pluie enregistré dans cette saison culturale, la préparation du terrain n'était intervenue que le 19-102012. Elle a consisté en un labour mécanique effectué avec le tracteur du centre de recherche (INERA). Cette

\section{RESULTATS}

Comparaison des effets de deux formes d'apport du bat-guano à ceux d'un engrais minéral sur la culture de niébé.

Paramètres de croissance : La comparaison des effets du bat-guano (formes dissoute et non dissoute) à opération à été suivie par un hersage effectué à la houe pour niveler le terrain et les travaux de délimitation du terrain. Le bat-guano était enfoui en fumure de fond avant le semis entre 10 à $15 \mathrm{~cm}$ le 28/10/2012, soit 4 semaines avant le semis. Le DAP a été épandu 2 semaines après le semis soit 12 décembre 2012. Le semis de niébé est intervenu le 2811-2012 à l'écartement de $(60 \times 20) \mathrm{cm}$ à raison de 2graines par poquet. Le regarnissage des vides a eu lieu le 05-12-2012 soit une semaine après le semis. Le sarclage à été effectué le 11/12/2012. Tout au long de cet essai ; nous avons eu à prendre des mensurations sur les paramètres végétatifs ci-après: le taux de levée, la hauteur de plants, le diamètre au collet, le nombre de ramifications. En ce qui concerne les paramètres de production, les données ci-après, ont été enregistrées : le nombre de gousses par plant, le nombre de graines par gousse, le poids de 100 graines, la production parcellaire, le rendement en tonnes/ha. Ces données ainsi recueillies, ont été soumises aux analyses statistiques avec les logiciels statistix 8.0. Ensuite, elles ont été soumises à l'analyse de variance pour tester la différence entre traitements. Le test HSD de Tukey a été utilisé pour comparer deux à deux les moyennes.

ceux d'un engrais minéral au niveau de paramètres de croissance de la culture de niébé, a donné les résultats repris au tableau 2.

Tableau 2 : Effets de deux formes du bat-guano et d'un engrais minéral (DAP) sur les paramètres croissance

\begin{tabular}{lcccc}
\hline Paramètres & Taux de levée $\%$ & $\begin{array}{c}\text { Diamètre au collet } \\
\text { (cm) }\end{array}$ & $\begin{array}{c}\text { Hauteur de plants } \\
\text { (cm) }\end{array}$ & $\begin{array}{c}\text { Longueur de } \\
\text { feuilles }\end{array}$ \\
\hline Traitements & & & & \\
\hline BD & $74.20 \mathrm{a}$ & $7.96 \mathrm{a}$ & $36.90 \mathrm{a}$ & $5.33 \mathrm{a}$ \\
BND & $70.03 \mathrm{a}$ & $8.20 \mathrm{a}$ & $37.00 \mathrm{a}$ & $5.00 \mathrm{a}$ \\
EM & $74.53 \mathrm{a}$ & $8.86 \mathrm{a}$ & $41.43 \mathrm{a}$ & $5.00 \mathrm{a}$ \\
$1 / 2(\mathrm{BD}+\mathrm{EM})$ & $70.70 \mathrm{a}$ & $7.86 \mathrm{a}$ & $41.20 \mathrm{a}$ & $4.00 \mathrm{a}$ \\
$1 / 2(\mathrm{BND}+\mathrm{EM})$ & $78.70 \mathrm{a}$ & $8.53 \mathrm{a}$ & $43.46 \mathrm{a}$ & $4.00 \mathrm{a}$ \\
TO & $62.40 \mathrm{a}$ & $7.90 \mathrm{a}$ & $39.06 \mathrm{a}$ & $5.66 \mathrm{a}$ \\
Décision & $\mathrm{NS}$ & $\mathrm{NS}$ & $\mathrm{NS}$ & $\mathrm{NS}$ \\
CV (\%) & & 8.03 & 12.09 & 17.18 \\
\hline
\end{tabular}

Les moyennes portant la même lettre, ne sont pas différentes au seuil de $5 \%$ pour le test HSD de Tukey. Nous l'observons bien pour ces paramètres de croissance, il $\mathrm{n}$ y a pas de différence significative entre les traitements qui ont eu les mêmes effets sur ces variables. Statistiquement, tous les traitements ont un effet similaire sur le taux de levée, le diamètre au collet, 


\section{Mulambuila et al. J. Appl. Biosci. Étude comparative de quelques fertilisants (Bat-guano et DAP) sur le rendement du niébé (Vigna unguiculata, L. Walp.) dans la région de Gandajika (RDC)}

la hauteur de plants, et le nombre de ramifications bien qu'il y ait quelques légères différences arithmétiques.

Paramètres de production : La comparaison des effets du bat-guano (sous ses deux formes) à ceux de l'engrais minéral au niveau des paramètres de production, a donné sur la culture de niébé, les résultats repris au tableau 3.

Tableau 3 : Effets de deux formes du bat-guano et de l'engrais minéral sur les paramètres de production

\begin{tabular}{lcccc}
\hline Paramètres & $\begin{array}{c}\text { Nombre de } \\
\text { gousse/pl }\end{array}$ & $\begin{array}{c}\text { Nombre de } \\
\text { graines/gousse }\end{array}$ & $\begin{array}{c}\text { Production } \\
\text { Par parcelle (kg) }\end{array}$ & $\begin{array}{c}\text { Rendement } \\
\text { (en tonne/ha) }\end{array}$ \\
\hline BD & $15 \mathrm{~b}$ & $13.66 \mathrm{a}$ & $0.550 \mathrm{a}$ & $0.916 \mathrm{a}$ \\
BND & $13.66 \mathrm{a}$ & $14.66 \mathrm{a}$ & $0.430 \mathrm{a}$ & $0.730 \mathrm{a}$ \\
EM & $15.00 \mathrm{a}$ & $14 \mathrm{a}$ & $0.470 \mathrm{a}$ & $0.780 \mathrm{a}$ \\
\hline 1/2(BD+EM) & $15.33 \mathrm{a}$ & $10.33 \mathrm{a}$ & $0.500 \mathrm{a}$ & $0.840 \mathrm{a}$ \\
\hline 1/2(BND+EM) & $11.33 \mathrm{a}$ & $12.66 \mathrm{a}$ & $0.420 \mathrm{a}$ & $0.730 \mathrm{a}$ \\
TO & $11 \mathrm{a}$ & $10 \mathrm{a}$ & $0.390 \mathrm{a}$ & $0.660 \mathrm{a}$ \\
Décision & $\mathrm{NS}$ & $\mathrm{NS}$ & $\mathrm{NS}$ & $\mathrm{NS}$ \\
CV (\%) & 17.05 & 19.35 & 17.90 & 17.75 \\
\hline
\end{tabular}

Les paramètres portants la même lettre ne sont pas différentes au seuil de 5\% par le test HSD de Tukey. Pour tous ces paramètres de production, il n'y a pas de différence significative entre les traitements qui ont eu

\section{DISCUSSION}

Au regard de résultats enregistrés sur les paramètres de croissance de la culture de niébé ayant fait l'objet de cet essai, nous notons que dans une saison culturale où les signes de phénomènes de changements climatiques ont été remarquables par le retard de pluies, et leur irrégularité. Le semis à été effectué avec un grand retard au mois de novembre au lieu de septembre ou octobre. Le volume d'eau (de pluie) enregistré au mois de décembre et de janvier a été excessif pour la culture de niébé, on a observé un grand développement végétatif qui s'est réalisé au détriment de la production en gousses (Anonyme, 1998). Les effets de fumures soumises à l'épreuve n'ont pas été notables à cause du volume excessif de

\section{CONCLUSION ET SUGGESTIONS}

Cette recherche a été intitulée: Comparaison de l'apport du bat-guano au Diammonium de phosphate (DAP) sur le rendement de la culture de niébé (Vigna unguiculata, L., Walp.) dans la région de Gandajika au de le RD. Congo. Rappelons les objectifs : déterminer entre les deux formes d'apport du bat-guano celle qui convient en efficacité sur la culture de niébé en supposant que la dissolution préalable du bat-guano contribuerait à la libération des éléments minéraux, ce qui faciliterait leur absorption rapide par la culture. les mêmes effets sur les variables. Statistiquement, tous les traitements ont eu les mêmes effets sur les différents paramètres de production.

l'eau. Les différentes fumures ont eu les mêmes effets sur les paramètres de croissance. Le facteur eau (volume de pluies et sa répartition tout au long de la saison culturale) n'ayant pas été à son optimum, n'a pas permis en toute vraisemblance aux autres facteurs dont les éléments minéraux de s'exprimer rationnellement sur les paramètres de croissance. La pluie a constitué un facteur limitant, les résultats enregistrés le montrent bien clairement. Pour ce qui concerne les paramètres de production, la situation a été similaire à celle observée avec les paramètres végétatifs; ceci, serait dû aux raisons sus-évoquées (mauvaise répartition de pluies) qui aurait eu une influence négative sur l'évolution ces paramètres

Déterminer aussi entre les formes, dissoute et non dissoute, celle qui serait un bon fertilisant organique, en comparaison avec le DAP, engrais minéral composé de deux éléments de base $\mathrm{N}$ et $\mathrm{P}$ comme dans le batguano. Les résultats obtenus indiquent bien que les deux formes ont les mêmes effets sur la culture de niébé. Ces résultats corroborent à ceux enregistrés par Kalambaie et Nkongolo , 2013; Hadas et Rosenberg, 1992. Les changements climatiques pouvant bien se traduire par le manque de pluies tout comme leur 
excès, ont un impact négatif sur la croissance et le rendement de cultures dans la mesure où cette irrégularité ne suit pas le cycle de développement de cultures. On peut bien observer le déficit en eau, au moment ou la culture en a besoin et l'excès quand la plante n'en a pas vraiment besoin. En suivant le retour de pluies pour procéder au semis, il peut bien arriver qu'une culture s'étale sur deux saisons culturales. Devant les phénomènes de changement climatiques, la recherche devra s'orienter non seulement vers l'obtention de variétés résilientes, mais aussi de cycle court, pour limiter l'irrégularité de pluies. Dans le cadre

\section{BIBLIOGRAPHIES}

Anonyme, 1998.: Monographie de la Province du Kasaï Oriental. Programme National de Relance du Secteur Agricole Rural (MNSAR) 997 - 2001 Programme des Nations Unies pour le Développement (PNUD). Agence de Nations Unies pour les Services aux Projets (UNOPS) Kinshasa. 277p.

Anonyme, 2003.: Rapports annuel du Territoire de Gandajika. Province du Kasaï Oriental, République Démocratique du Congo.2-5pp.

Ayoola, O.T. and Makinde, E.A. (2008): Farming Systems Research and Extension Programme, Institute of Agricultural Research and Training, Obafemi. in African Journal of Plant Science Vol. 2(3), 019 - 022 pp.

Bado, B.V., D. Dakyo, A. N'Daye gamine et M.P. les cas. 1993.: Effet de la dolomie sur la production et les propriétés chimiques d'un sol ferrallitique. Agrosol 6(2) : 22-24pp.

Bado, B.V., Bationo, A., Lompo, F, Cescas, M.P. and Sedogo, M.P. (2007). : Mineral fertilizers, organic amendments and crop rotation managements for soil fertility maintenance in the Guinean zone of Burkina Faso (West Africa). In: Bationa A., Waswa B., Kihara J, Kimetu $\mathrm{J}$ (eds). Advances in integrated soil fertility management in Sub. Saharan Africa: Challenges and opportunities, Springer, The Netherlands 171-177pp

Bagayoko, M.S., Mason, C., Traoré, S. and Eikert, K.M. (1996): Pearl millet/cowpea cropping system yield and soil nutrient levels. African crop science journal4 (4): 453-462pp.

Bartoli, F., Burtin, G., Guerif, J.(1992) Influence of organic matter on aggregation in oxisols rich in gibbsite or goethite": clay dispersion, de cette étude, le Bat-guano peut être recommandé aux paysans producteurs étant donné sa richesse en éléments nutritifs, son accessibilité, son coût moins onéreux comparativement aux engrais minéraux (DAP). La contrainte de sa disponibilité est contournée par l'élevage de Chauve-souris qui produisent suffisamment ce fertilisant. En rapport, la GIFS, bien que le facteur eau n'ait pas permis au Bat-guano d'enregistrer les résultats escomptés. Son utilisation peut bien se justifier pour minimiser la quantité d'engrais minéraux à utiliser.

aggregate strength and water stability, Geoderma 54: 259-274pp

Bationo, A., Lompo, F., Koala, S., Nandwa, S. and Bekunda, M. (1997). Nutrient balance studies and available technologies to combat land degradation in Africa. IFDCI ICRISAT.

Bui, E.N., Mermut, A.R., and Santos, M.C.D (1989) Microscopic and ultramicroscopic porosity of an oxisol as determined by image analysis and water retention. Soil Science Society of America Journal 53: 661-665pp.

Buresh, J.R. and Niang, IA (1997) Tithonia diversifolia as a green manure: awareness expectations, and reality, Agroforestry Forum, 29-30pp

Coullibaly, A., Bagayogo, M.S., Traoré, S. and Mason, C. (1998) Crop residue management and system influence on pearl millet and cowpea yield. INTSORMIL grant. Contribution of $1 \mathrm{er}$ and University of Nebraska, Lincoln, NE.

Culot, J.P. et H.Laudelout.1957. Rétrogradation et l'utilisation des engrais phosphatés dans les sols du Congo Belge. Pédologie 7: 162$168 \mathrm{pp}$.

Deckers, J. (1993) Soil fertility and environnement problems in different ecological in Subsaharan Africa In: Role of Plant Nutrients of Sustainable Food Production in Sub-Saharan Africa.

Falisse H. et Lambert J., 1995. La fertilisation Minérale et Organique dans l'Agronomie Moderne, bases Physiologiques et Agronomiques de la production Végétale. Éditeurs: El Hassani, Tayeb Ameziane, M. Guillou et E. Persoons. AUPELF- UREF. Hatier. France, Paris, pp384397.

FAO, 1999. Synthèse agronomique des essais de fertilisation dans la République Démocratique 
du Congo, CD-ROM, division de la mise en valeur des terres et des eaux FAO? Viale delle terme di Caracalla, Rome.

Hadas, A., et Rosenberg, R., 1992. Guano as a nitrogen source for fertigation in organic farming, fertilizer Reseach, Vol 31, Issue 2, $209-214 p p$.

INERA, 2007: Programme légumineuse, fiches d'identification des variétés améliorées $d u$ niébé, Ngandajika.

INERA, 2007. Rapport annuel Scientifique, 53p.

Ingram, J. (1990) The role of trees in maintaining and improving soil productivity: a review of the literature. Technical paper $n^{\circ} 279$. Commonwealth, Science Council, 39p.

Kalambaïe Bin Mukanya M. et Nkongolo Mulambuila M., 2013. Effetsb compares de l'apport du Batguano sous forme dissolute à la forme nondissoute sur la culture du Maïs dans la région de Mbujimayi, (RDC), Saison B in Les Cahiers de l'Université Officielle de Mbujimayi, vol 1 , $n^{\circ} 5,17-23 p p$

Kang, BT, Wilson, GR. and Lawson, TL (1984) Alley cropping, a stable alternative to shifting cultivation. IITA Publication, 22p.

Muyayabantu M., 2013. Potentialités fertilisantes biologiques Locales en vue de l'amélioration d'un oxisol en Afrique Sub-saharien, These de doctorat, FSA/UOM.

Neufeld, H. (1999) Physical and Chemical properties of selected oxisols in the Brazilian Cerrados pp 37-50. In: Thomas, R. and Ayarza, M.A. (Eds) sustainable land management for the oxisols of the latin American savannas: Dynamics of soil organic matter and indicators of soil quality. CIAT publication $\mathrm{N}^{\circ} 312$ Cali, Colombia.

Ndembo, 2011. Manuel de formation des parties prenantes du Secteur Agricole sur la résilience du Secteur Agricole aux changements climatiques, 2011 Kimpese.

Nziguheba, G., Palm, C.A., Buresh, R.J. and Smithson, P.C. (1998). Soil phosphorus fractions and adsorption as affected by organic and inorganic sources. Plant and soil. 198: $159-$ $168 p p$.

Palm C.A., Myers, R.J.K., Nndwa, S.M. (1997) Combined use of organic and inorganic nutrient sources for soil fertility replenishment. In: Buresh, R. (Ed), Replenishing soil fertility in Africa. SSSA special publication Number 51 , SSSA, USA 193 - 217pp.

Rishirumuhirwa, T.E. ; Birasa, C., Bigura L., Lunze et M. Kurayum, 1989. Pedological study of eight sites for fertilizers trails in Economic Community of Great Lakes Countries C.E.P.G.L. (Moso, Mashitsi, Rubona, Karama, Yangambi, Mulungu, Gandajika, M'Vuazi, Gitega, République de Burundi, IRAZ (Institut de Recherche Agronomique et Zootechnique de la CEPGL) 110p.

San chez PA, Logan, T.L (1992). Myths and Science about the Chemistry and fertility of soils in the tropics In: Myths and science of Soils of the tropics Lal, R. and Sanchez, P.A. (eds) SSSA Special Publication $\mathrm{N}^{\circ} 29$ SSSA and ASA, Madison Wisconsin.

Wong, M.T.F., Akyeampong, A., Nortcliff, S., Rao, M.R. and Swift, R.S. (1995) Initial responses of maize and beans to decreased concentrations of monomeric inorganic aluminum with application of manure or tree prunings to an oxisol in Burundi. Plant soil 171: 275.

Young, A. (1987) The potential of agroforestry for soil conservation. Part II. Maintenance of fertility. ICRAF Working paper, 43p. 